\title{
USO DA EQUAÇÃO LOGÍSTICA NO ESTUDO DE DOSE-RESPOSTA DE GLYPHOSATE E IMAZAPYR POR MEIO DE BIOENSAIOS ${ }^{1}$
}

\author{
ADAILSON P. DE SOUZA ${ }^{2}$, FRANCISCO A. FERREIRA ${ }^{3}$, ANTÔNIO A. DA SILVA ${ }^{3}$, ANTÔNIO A. CARDOSO ${ }^{3}$ e HUGO A. RUIZ
}

\section{RESUMO}

O uso de bioensaios constitui uma das técnicas mais comuns de se avaliar o potencial de um herbicida e identificar e quantificar seus resíduos no solo ou na água. Nesses trabalhos, é fundamental conhecer os efeitos que algumas variáveis exercem sobre o material biológico e, quase sempre, é possível relacioná-las por meio de uma expressão matemática. Um dos problemas na obtenção das expressões consiste em encontrar aquela que melhor represente a relação doseresposta. $\mathrm{O}$ presente trabalho apresenta um estudo comparativo da equação logística $\left(\mathrm{y}=\mathrm{a} /\left(1+(\mathrm{X} / \mathrm{b})^{\mathrm{c}}\right)\right)$ com as equações polinomiais normais de $1^{\circ}, 2^{\circ} \mathrm{e}$ $3^{\circ}$ graus. Na avaliação do modelo mais adequado para o estudo de dose- resposta com o glyphosate e o imazapyr, foram considerados alguns critérios de ordem teórica e de aplicação prática, sendo possível concluir que: a) a função logística apresenta a estimativa de seus parâmetros significativa para o herbicida imazapyr e de razão biológica justificável, para ambos os herbicidas; b) as características de biomassa seca total (BST), da parte aérea (BSA), da raiz (BSR) e do comprimento da raiz (CR) são adequadas para estudos sobre a relação dose-resposta; c) a estimativa do $I_{50}$ é variável com a função e a característica avaliada; e d) o tomateiro é mais sensível ao imazapyr.

Palavras chave: Estatística, herbicida.

\begin{abstract}
The bioassay use is one of the more common techniques for evaluating an herbicide potential as well as identifying and quantifying its residues in soil or in water. In these studies it is very important to know the effects exerted by some variables on the biological material and almost always it is possible to relate them by a mathematical expression. A problem in obtaining the expressions is to find that one which better represents the dose-response relation. This work presents a comparative study between the logistic equation $\left[\mathrm{y}=\mathrm{a} /\left(1+(\mathrm{X} / \mathrm{b})^{\mathrm{c}}\right)\right]$ and the normal polynomial equations of $1^{\circ}, 2^{\circ}$ and $3^{\circ}$ degrees. In
\end{abstract}

ABSTRACT

\section{Logistic equation use in studying the dose-response of glyphosate and imazapyr by using bioassays}

evaluating the more adequate model for the dose-response study with glyphosate and imazapyr, some criteria of theoretical order and practical application were considered, and it was concluded that: a) the logistic function presents a significative estimate of its parameters for herbicide imazapyr and a justifiable biological reason for both herbicides; b) the characteristics of total dry biomass (BST), aerial part (BSA), root (BSR) and root length (CR) are adequate for the doseresponse relation studies; c) the $\mathrm{I}_{50}$

\footnotetext{
${ }^{1}$ Recebido para publicação em 28/05/98 e na forma revisada em 16/08/99.

${ }^{2}$ Prof. Adjunto do Depto. de Solos e Eng. Rural da UFPB, CEP 58397 -000, Areia - PB.

${ }^{3}$ Prof. Adjunto do Depto. de Fitotecnia da UFV, CEP 36571-000, Viçosa - MG.

${ }^{4}$ Prof. Adjunto do Depto. de Solos da UFV, CEP 36571 -000, Viçosa - MG.
} 
estimate is variable with the function and the evaluated characteristic; d) the tomato is more sensible to imazapyr.

Key words: Statistics, herbicide.

\section{INTRODUÇÃO}

Uma das técnicas mais comuns de se avaliar a eficácia de um herbicida é por meio do uso de bioensaios. Bioensaio é um experimento conduzido para estimar o potencial de um herbicida pela análise da reação que se segue, após sua aplicação sobre um organismo vivo. A relação entre a dose do herbicida e a resposta da planta é de fundamental importância no entendimento de sua eficácia e de seu mecanismo de ação. A compreensão desse relacionamento é essencial para o planejamento e interpretação dos trabalhos no campo, em casa de vegetação ou no laboratório. $\mathrm{O}$ bioensaio clássico, freqüentemente usado para identificar e quantificar um herbicida no solo ou na água, emprega uma única curva de dose-resposta padrão. Esta curva-padrão é usada para estimar a quantidade do herbicida em uma amostra, com base na resposta do bioindicador na própria amostra (Nyffeler et al., 1982).

Do ponto de vista estatístico, os bioensaios são experimentos planejados para estimar o potencial de um ou mais estímulos relativos a um padrão, usando a informação proporcionada pela resposta mensurada sobre o material biológico (Finney, 1979). A análise de variância é o ponto central para a maior parte das aplicações dos métodos estatísticos nas análises de experimentos com bioensaios e de fundamental importância na análise de regressão. Nos trabalhos com herbicidas, em muitas situações, é fundamental conhecer os efeitos que algumas variáveis (dose, época de aplicação e de coleta, etc.) exercem, ou que parecem exercer, sobre o material biológico, e quase sempre é possível relacioná-las, por meio de uma expressão matemática. Observa-se, portanto, que a aplicação de métodos estatísticos apropriados aos dados de um bioensaio pode levar o pesquisador a obter uma maior confiabilidade nos resultados obtidos. Por outro lado, percebe-se também que, na prática, poucos trabalhos parecem ter submetido seus dados a uma criteriosa análise estatística, o que acarreta, muitas vezes, perdas de informações ou inadequação de interpretações do fenômeno em estudo.

O maior problema na avaliação da relação dose-resposta é a obtenção de modelos que melhor representem a relação. A primeira solução adotada pelos pesquisadores foi linearizar os dados com transformações logarítmicas, exponenciais, logísticas ou probits (Berkson, 1953; Nelder, 1961; Finney, 1971; Finney, 1976). Tais transformações, entretanto, usualmente apresentam limitada razão biológica. Acredita-se que a maior parte das curvas de dose-resposta é não-linear. Um modelo é dito não-linear quando pelo menos um de seus parâmetros aparece nãolinearmente, ou seja, o parâmetro aparece como expoente, ou multiplicado ou dividido por outro parâmetro, ou em funções transcendentes como exponenciais, logarítmicas e trigonométricas. Os cálculos necessários para desenvolver a equação que poderá predizer o melhor ajuste da curva de regressão não-linear, em geral, são deduzidos a partir de suposições teóricas (Cordeiro \& Paula, 1989). A razão disso é a complexidade apresentada na solução analítica para o problema de estimação dos parâmetros, principalmente quando intrinsecamente não-lineares. Segundo Draper \& Smith (1981), quando informações teóricas, com relação ao modelo, levam a um modelo intrinsecamente não linear, deve-se preferir tal modelo, sempre que possível. Nesses modelos, o problema de estimação depende essencialmente da utilização de métodos de otimização não-lineares, sendo os algoritmos de Gauss-Newton, Marquardt \& Simplex os mais usados (Nelder \& Mead, 1965; Motulsky \& Ransnas, 1987).

Na década de 90, a ampla disponibilidade de microcomputadores de alto desempenho e pacotes estatísticos dos mais diversos (SAS, SAEG, Statistica, TBC, etc.), tem possibilitado uma generalização da técnica de análise não- 
linear, o que tem implicado em escolha de modelos com comportamento dos mais variados e, em alguns casos, inadequados para as variáveis em estudo. Acrescenta-se, ainda, que o uso de variados modelos para um mesmo fenômeno tem dificultado a comparação entre resultados de trabalhos científicos.

O presente trabalho tem por objetivo fazer um estudo comparativo da equação logística com as equações de regressão mais comumente usadas nos estudos de dose-resposta com herbicidas.

\section{MATERIAL E MÉTODOS}

\section{Experimentos}

Dois experimentos, um com o glyphosate e outro com o imazapyr, foram conduzidos em casa de vegetação, utilizando-se o tomateiro (Lycopersicon esculentum Mill var. Santa Clara) como bioindicador. Inicialmente, as sementes foram colocadas para germinar em areia de quartzo. Cinco dias após a germinação (início da formação do primeiro par de folhas definitivas), foram transplantadas duas plantas para cada vaso de polietileno com 1,5 L de solução nutritiva de Hoagland \& Arnon (1938), com redução de 50\% da concentração de fósforo. Após $12 \mathrm{hs}$, fez-se a aplicação dos tratamentos, correspondentes às seguintes doses dos herbicidas na solução nutritiva: $0 ; 32 ; 64 ; 128 ; 256$ e $512 \mu \mathrm{g} \mathrm{L}^{-1}$, para o glyphosate e $0 ; 1,775 ; 3,55 ; 7,1 ; 14,2$ e $28,4 \mu \mathrm{g} \mathrm{L}-$ ${ }^{1}$, para o imazapyr. As doses em escala geométrica foram obtidas após ter sido delimitado o extremo superior $\left(d_{s}\right)$, num pré-ensaio experimental. As interpolações das doses entre a testemunha $\left(d_{0}\right)$ e o extremo superior foram obtidas por:

$d_{n}=\frac{d_{s}}{q^{N-(n-1)}}$,

em que:

$\mathrm{n}=1,2, \ldots, \mathrm{N}$

$\mathrm{N}=$ número de termos a interpolar; $\mathrm{e}$

$\mathrm{q}=$ razão da progressão geométrica, para $\mathrm{q} \geq 2$.
Os vasos foram mantidos sob aeração constante e o $\mathrm{pH}$ corrigido diariamente para 5,7 \pm 0,02 . Vinte dias após a aplicação dos herbicidas, efetuou-se a coleta das plantas, determinando-se o comprimento das raízes. Após a separação da parte aérea das raízes, as mesmas foram acondicionadas separadamente em sacos de papel e colocadas para secar em estufa com circulação de ar, a $65^{\circ} \mathrm{C}$, até peso constante. Ao final da secagem do material (aproximadamente $72 \mathrm{~h}$ ), procedeu-se a pesagem e os resultados analisados estatisticamente.

O delineamento estatístico utilizado foi o inteiramente casualizado com seis tratamentos, correspondentes às doses do herbicida, e cinco repetições. As características avaliadas por planta (biomassa seca total, BST; da parte aérea, BSA; das raízes, BSR; e comprimento das raízes, $\mathrm{CR}$ ) permitiram a estimativa de uma variância ponderada entre a variância experimental e a de amostragem, obtida por:

$$
\mathrm{QMR}_{\text {ponderado }}=\frac{\mathrm{I}(\mathrm{J}-1) \mathrm{QMR} \text { experimental }+\mathrm{IJ}(\mathrm{K}-1) \mathrm{QMR} \text { amostragem. }}{\mathrm{I}(\mathrm{JK}-1)}
$$

com I(JK-1) G.L., em que:

QMR = quadrado médio do resíduo;

$\mathrm{I}=\mathrm{n}^{\mathrm{o}}$ de tratamentos;

$\mathrm{J}=\mathrm{n}^{\mathrm{o}}$ de vasos; $\mathrm{e}$

$\mathrm{K}=\mathrm{n}^{\mathrm{o}}$ de plantas por vaso.

\section{A curva de dose-resposta}

A relação da $\mathrm{BST}$, da BSA, da BSR e do $\mathrm{CR}$ com as doses do herbicida foram analisadas por regressão, testando-se os modelos logísticos e polinomiais de $1^{\circ}, 2^{\circ}$ e $3^{\circ}$ graus.

O modelo logístico adotado, eq. 2 (Streibig et al., 1993), apresenta três parâmetros, cujos estimadores não têm fórmula explícita e as estimativas foram obtidas por meio de técnicas numéricas, denominadas de otimização não linear, com o método do algoritmo de Marquardt, utilizado para resolver o problema de mínimos quadrados (Marquardt, 1963). Esse modelo fica entre duas assíntotas horizontais, que são o eixo 
das abcissas e a reta de ordenada constante e igual a "a”". O parâmetro "a”, que é a distância entre as duas assíntotas, é denominado "nível de saturação", correspondente à resposta do bioindicador na dose zero. O parâmetro " $a_{1}$ " é o ponto de inflexão da curva, que corresponde ao $\mathrm{I}_{50}$ (concentração do produto que inibe em 50\% o crescimento do bioindicador). O parâmetro " $\mathrm{a}_{2}$ " descreve o declive da curva em torno do $I_{50}$. Assim:

$y=\frac{a_{0}}{1+\left[\frac{x}{a_{1}}\right]^{a_{2}}}$

Os modelos polinomiais de $1^{\circ}, 2^{\circ}$ e $3^{\circ}$ graus (eq. 3, 4 e 5, respectivamente) seguiram a forma polinomial geral, $\nabla=a_{0}+a_{1} X+\ldots+a_{n} X^{n}$, em ordem crescente de grau:

$\hat{y}=a_{0}+a_{1} x$

$\hat{y}=a_{0}+a_{1} x+a_{2} x^{2}$

$\hat{y}=a_{0}+a_{1} x+a_{2} x^{2}+a_{3} x^{3}$

As estimativas dos parâmetros das equações polinomiais foram obtidas de acordo com o método de mínimos quadrados, utilizandose a notação matricial na resolução dos sistemas (Hicks, 1973).

Tanto para a análise de variância quanto para a estimativa dos parâmetros da regressão dos modelos, foi utilizado o pacote estatístico SAEG (SAEG, 1997).

A concentração de cada herbicida, necessária para a inibição de $50 \%$ do crescimento da planta bioindicadora $\left(\mathrm{I}_{50}\right)$, foi obtida mediante fórmulas deduzidas a partir dos modelos de regressão. As fórmulas gerais usadas nessa determinação para os modelos logístico, linear e quadrático (eq. 6, 7 e 8, respectivamente) foram:

$\mathrm{I}_{\mathrm{i}}=\mathrm{a}_{1}\left(\frac{\mathrm{i}}{100-\mathrm{i}}\right)^{\mathrm{i} / \mathrm{a}_{2}}$

$$
I_{i}=\frac{a_{0}}{a_{1}}\left(\frac{i}{100}-1\right)
$$

$$
\mathrm{I}_{\mathrm{i}}=\frac{-\mathrm{a}_{1} \pm \sqrt{\mathrm{a}_{1}^{2}-4 \mathrm{a}_{2}\left(\mathrm{a}_{0}-\frac{\mathrm{i} \mathrm{a}_{0}}{100}\right)}}{2 \mathrm{a}_{2}}
$$

em que i é o percentual de redução de crescimento.

Em razão da complexidade apresentada na resolução do valor de $I_{i}$ da equação de $3^{\circ}$ grau, cuja expressão obtida é tão extensa que sua utilização resulta ser impraticável para o cálculo efetivo, os valores de $I_{50}$ foram obtidas pelo método de resolução gráfico-interativo.

$\mathrm{Na}$ avaliação do modelo mais adequado para o estudo de dose-resposta, foram considerados alguns critérios de ordem teórica e de aplicação prática. Nos critérios de ordem teórica verificaram-se: a contribuição de cada parâmetro para a soma de quadrados da regressão, pelo teste F parcial (Motulsky \& Ransnas, 1987); a significância, pelo teste $F$, da falta de ajustamento do modelo (FA) e grau de ajustamento do modelo $\left(\mathrm{R}^{2}\right.$, coeficiente de determinação); a dispersão relativa, medida pelo CV (Coeficiente de Variação) da regressão; e a significância, pelo teste $\mathrm{t}$, das estimativas dos parâmetros da regressão dos modelos polinomiais (Hoffmann \& Vieira, 1983).

Os critérios de aplicação prática compreenderam: a observação do comportamento das curvas com base em uma razão biológica justificável e a determinação do valor do $\mathrm{I}_{50}$.

\section{RESULTADOS E DISCUSSÃO}

Observa-se na Tabela 1, que não houve significância do resíduo experimental para as características de BST, BSA, BSR e CR no experimento com o glyphosate, e de BST e BSR no experimento com o imazapyr, demonstrando que não houve variação entre as médias das plantas dentro de cada vaso. 
TABELA 1. Resumo da análise de variância da biomassa seca total (BST), da parte aérea (BSA), das raízes (BSR) e comprimento das raízes (CR) do tomateiro (Lycopersicon esculentum Mill var. Santa Clara), sob doses crescentes de glyphosate e imazapyr.

\begin{tabular}{|c|c|c|c|c|c|c|}
\hline \multirow[t]{2}{*}{ Herbicida } & \multirow[t]{2}{*}{ Fonte de Variação } & \multirow{2}{*}{ G.L. } & \multicolumn{4}{|c|}{ Quadrado Médio } \\
\hline & & & BST & BSA & BSR & CR \\
\hline \multirow{17}{*}{ Glyphosate } & Parcela & 29 & 0,364757 & 0,279537 & 0,006709 & 489,0453 \\
\hline & (Dose) & (5) & $1,408814 * *$ & $1,051392 * *$ & $0,026309^{* *}$ & $2384,6040 * *$ \\
\hline & Linear & 1 & $6,531417 * *$ & $4,890427 * *$ & $0,118496^{* *}$ & $11496,0750 * *$ \\
\hline & Resíd. da regressão & 58 & 0,125727 & 0,097652 & 0,117114 & 79,8332 \\
\hline & Falta de ajustamento & 4 & 0,128162 & 0,091633 & 0,003263 & 81,7359 \\
\hline & Logístico & 2 & 3,357785 & 2,509315 & 0,062115 & 5602,6254 \\
\hline & Resíd. da regressão & 57 & 0,124703 & 0,097116 & 0,119068 & 86,3359 \\
\hline & Falta de ajustamento & 3 & 0,109499 & 0,079443 & 0,002439 & 205,9228 \\
\hline & Quadrático & 2 & 3,292869 & 2,460367 & 0,060987 & 5756,5449 \\
\hline & Resíd. da regressão & 57 & 0,126980 & 0,098834 & 0,119107 & 80,9353 \\
\hline & Falta de ajustamento & 3 & 0,152777 & 0,112075 & 0,003191 & 103,3098 \\
\hline & Cúbico & 3 & 2,241428 & 1,675789 & 0,041353 & 3860,1715 \\
\hline & Resíd. da regressão & 56 & 0,126774 & 0,098694 & 0,121197 & 81,1765 \\
\hline & Falta de ajustamento & 2 & 0,159893 & 0,114795 & 0,003744 & 121,2524 \\
\hline & Resíd. Experimental & 24 & 0,147246 & 0,118733 & 0,002625 & 98,3038 \\
\hline & Resíd. Amostragem & 30 & 0,108188 & 0,081589 & 0,002018 & 64,8028 \\
\hline & Resíd. Ponderado & 54 & 0,125547 & 0,098098 & 0,002288 & 79,6922 \\
\hline \multirow{17}{*}{ Imazapyr } & Parcela & 29 & 0,960899 & 0,703096 & 0,020826 & 770,2288 \\
\hline & (Dose) & (5) & $4,599214 * *$ & $3,329597 * *$ & $0,104418^{* *}$ & $4109,0420 * *$ \\
\hline & Linear & 1 & $18,346313 * *$ & $13,313465 * *$ & $0,402587 * *$ & $17152,8560^{* *}$ \\
\hline & Resíd. da regressão & 58 & 0,222912 & 0,163989 & 0,005102 & 104,4881 \\
\hline & Falta de ajustamento & 4 & $1,162440 * *$ & $0,833631 * *$ & $0,029875 * *$ & $848,0877 * *$ \\
\hline & Logístico & 2 & 11,322937 & 8,186738 & 0,258939 & 10141,1585 \\
\hline & Resíd. da regressão & 57 & $0,151392 * *$ & $0,113181 * *$ & $0,003169 * *$ & $51,4184 * *$ \\
\hline & Falta de ajustamento & 3 & 0,116733 & 0,091504 & 0,001403 & 87,6300 \\
\hline & Quadrático & 2 & 11,034165 & 7,934987 & 0,255857 & 10079,8520 \\
\hline & Resíd. da regressão & 57 & $0,161524 * *$ & $0,122015^{* *}$ & $0,003277 * *$ & $53,5695 * *$ \\
\hline & Falta de ajustamento & 3 & 0,309248 & 0,259338 & 0,003458 & 128,5011 \\
\hline & Cúbico & 3 & 7,481209 & 5,411863 & 0,170593 & 6772,8040 \\
\hline & Resíd. da regressão & 56 & 0,157707 & 0,117644 & 0,003334 & 51,6920 \\
\hline & Falta de ajustamento & 2 & 0,276224 & 0,206201 & 0,005155 & 113,3975 \\
\hline & Resíd. Experimental & 24 & 0,202917 & $0,155908^{*}$ & 0,003413 & $74,6425 * *$ \\
\hline & Resíd. Amostragem & 30 & 0,113637 & 0,081168 & 0,003152 & 29,2186 \\
\hline & Resíd. Ponderado & 54 & 0,153317 & 0,114386 & 0,003267 & 49,4067 \\
\hline
\end{tabular}

$*, * *$ : significativo a $5 \%$ e $1 \%$, respectivamente, pelo teste $\mathrm{F}$. 
Esses resultados indicam que, apesar de haver rigor na seleção das mudas e na condução dos experimentos, as mesmas ainda apresentaram elevada heterogeneidade, que não foi estimada pelo resíduo experimental, havendo, portanto, a necessidade de se testarem os efeitos dos tratamentos (doses) por meio do resíduo ponderado. Segundo Steel \& Torrie (1960), a estimativa da variação entre as subamostras ou unidades de amostragem, dentro de uma unidade experimental, é uma medida de homogeneidade da unidade, e essas variações dependem, sobretudo, das diferenças ambientais e, ou, genéticas de planta para planta, dentro de cada vaso.

Ainda na Tabela 1, constata-se efeito significativo para o modelo linear, em todas as características avaliadas, tanto para o experimento com o glyphosate, quanto para o imazapyr, e com falta de ajustamento significativo apenas no imazapyr. Hoffmann \& Vieira (1983) afirmam que a significância na falta de ajustamento (FA) implica em que o modelo testado não é apropriado, visto que o quadrado médio da falta de ajustamento está estimando um erro sistemático a mais que a estimativa de $\sigma^{2}$. Portanto, é sempre desejável que o FA dê um resultado nãosignificativo, como observado no experimento do glyphosate. No experimento com o imazapyr, tanto o modelo logístico quanto o quadrático mostraram-se significativos, com a FA nãosignificativo, indicando que tais modelos são mais adequados para descrever o fenômeno em estudo.

Nas Figuras 1, 2, 3 e 4, constata-se que os $\mathrm{R}^{2}$ dos modelos linear, logístico, quadrático e cúbico foram significativos em todas as características avaliadas nos experimentos com o glyphosate e o imazapyr. Tais resultados apresentam limitado significado no processo de escolha do melhor modelo, pois uma vez obtido significância no modelo de menor grau (linear), haverá elevada probabilidade de terem-se efeitos significativos com o aumento dos graus ou parâmetros do modelo. Isso ocorre, porque, nos modelos com comportamento aproximadamente normal, a inclusão de novas variáveis sempre acarretará melhor ajuste do modelo aos pontos observados. Segundo Gauston \& Venus (1981), modelos envolvendo maior número de parâmetros, usualmente, são mais flexíveis, possibilitando melhor ajuste.

Dentre as funções estudadas, segundo o critério do $R^{2}$, a função que apresentou o mais baixo ajuste foi a linear, com exceção apenas na variável $\mathrm{CR}$, para o glyphosate. Apesar de as funções quadrática e cúbica apresentarem ótimo ajuste, o significado biológico de seus parâmetros para algumas características, é de difícil interpretação, como se observa principalmente para o imazapyr, onde são obtidos valores negativos com o aumento das doses para as características BST, BSA, BSR e CR (Figuras 1, $2,3$ e 4$)$.

Nas características avaliadas para os dois produtos, a função logística apresentou os mais baixos valores de CV da regressão, sendo este ainda menor no imazapyr. Considerando-se que o CV mede a relação entre a medida de dispersão dos pontos observados em torno da curva, expressa por $s$ ( $\left.\sqrt{\mathrm{QM}_{\text {res. da regres. }}}\right)$, e o valor médio da variável dependente, $\bar{y}$, o resultado é tanto melhor, quanto menor for o $\mathrm{CV}$ da regressão. Assim, a função logística apresenta-se como a de melhor qualidade de ajustamento.

As curvas de biomassa seca e comprimento de raiz, correspondentes aos ajustes das diferentes funções matemáticas aos dados, demonstraram que a função logística é a que melhor representa o fenômeno em estudo. Nas doses iniciais, observam-se respostas aproximadamente constantes, seguindo-se a redução da resposta com o aumento das doses. Tais resultados obtidos pelo ajuste logístico refletem a baixa toxicidade dos herbicidas ao bioindicador, nas menores doses, com efeitos altamente tóxicos nas maiores doses. 


\section{Glyphosate}
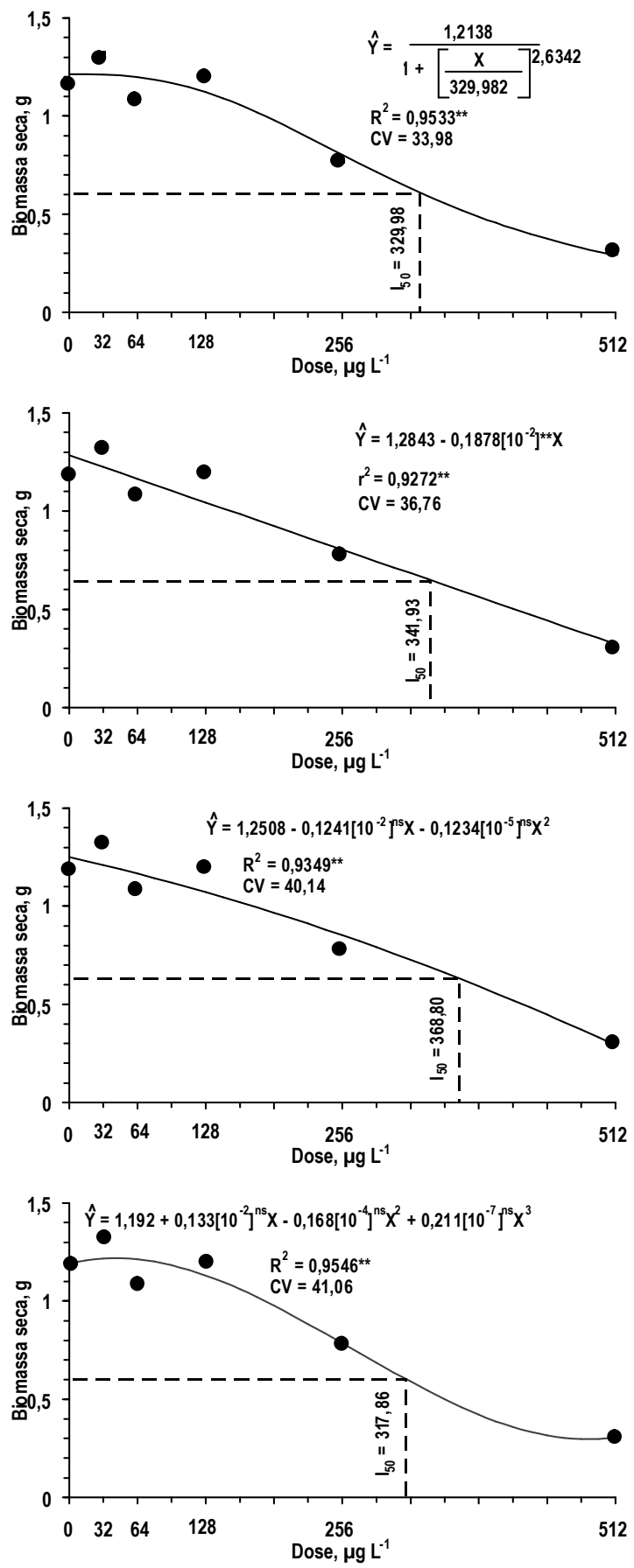

\section{Imazapyr}
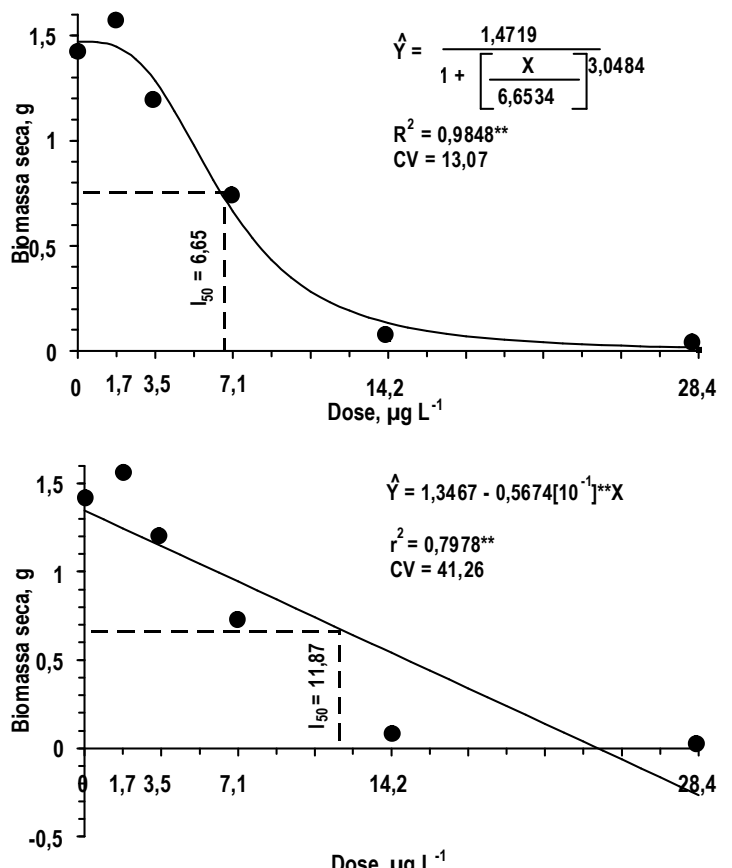

Dose, $\mu g \mathrm{~L}^{-1}$
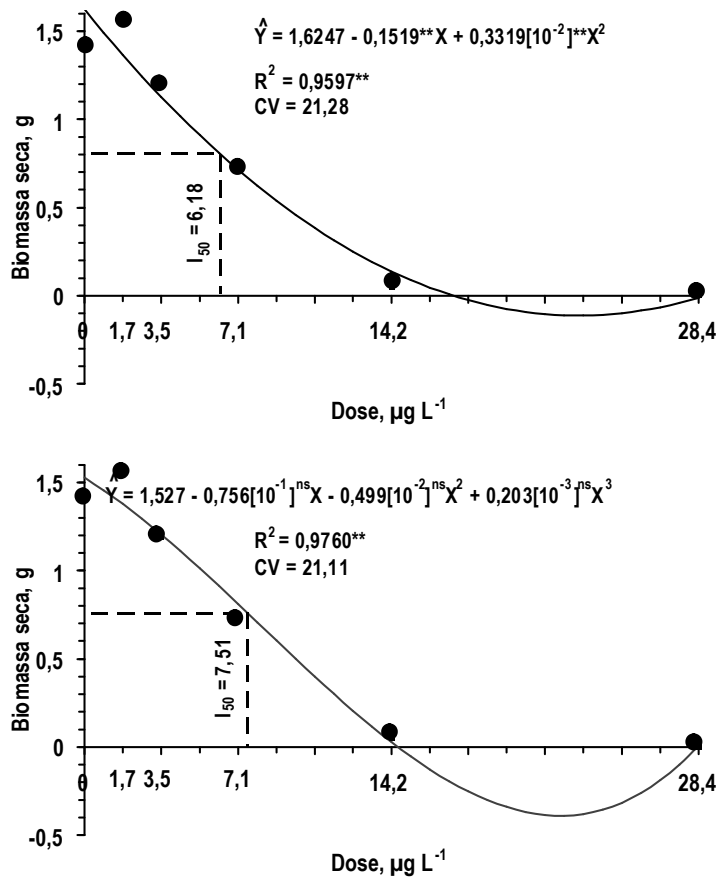

FIGURA 1. Curvas de dose-resposta da biomassa seca total (BST) do tomateiro (Lycopersicon esculentum Mill. var. Santa Clara), sob doses crescentes de glyphosate e imazapyr. 


\section{Glyphosate}
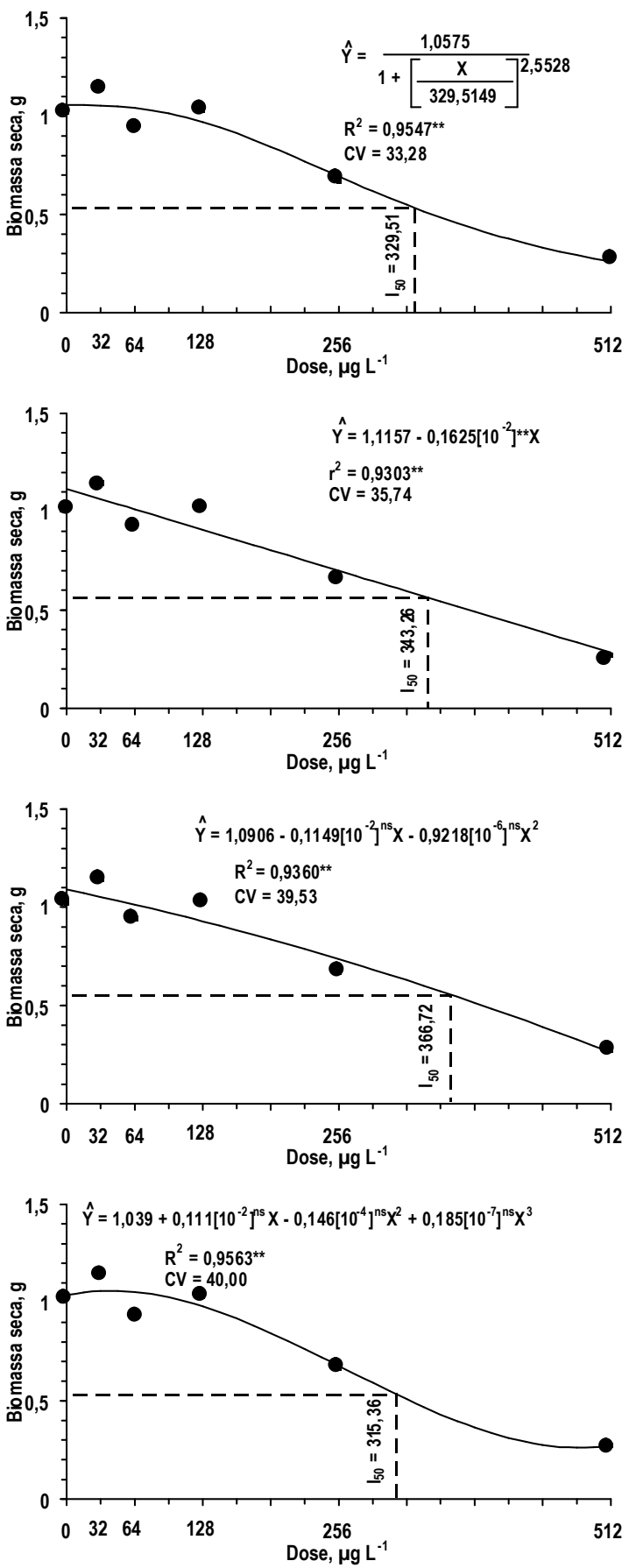

\section{Imazapyr}
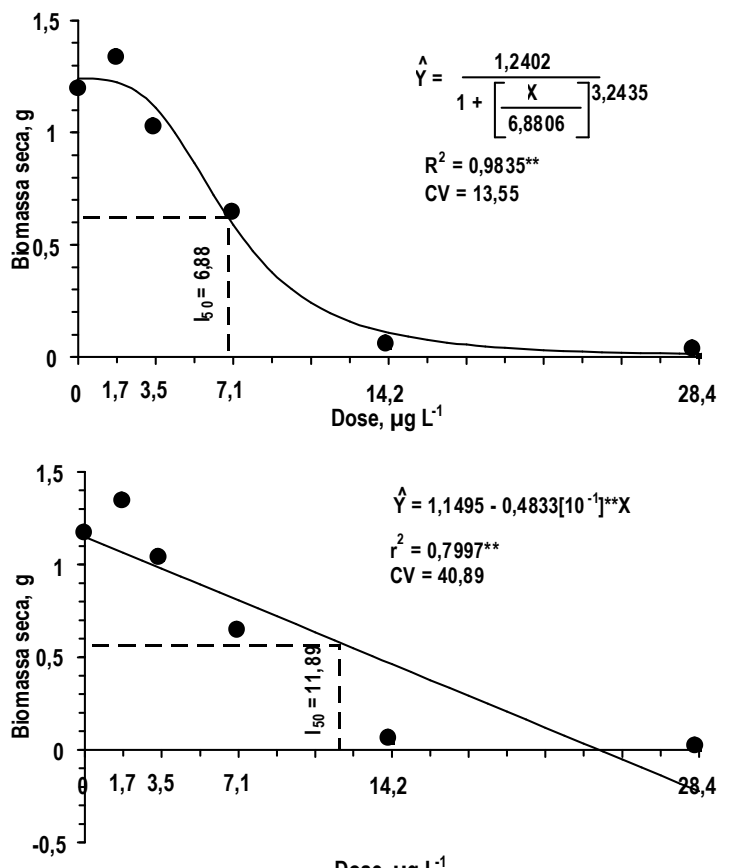

Dose, $\mu \mathrm{g} \mathrm{L}^{-1}$
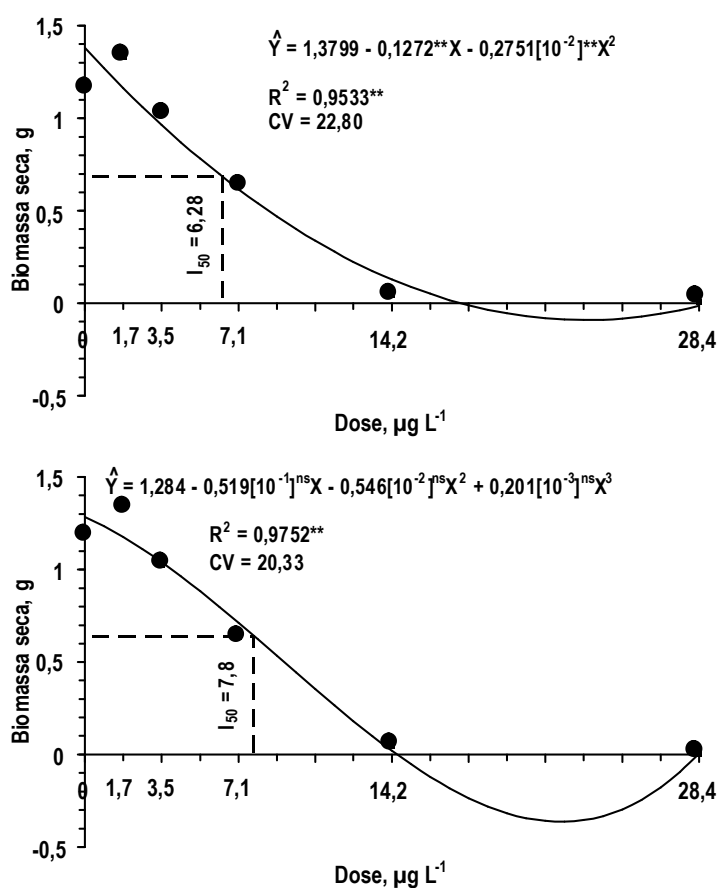

FIGURA 2. Curvas de dose-resposta da biomassa seca da parte aérea (BSA) do tomateiro (Lycopersicon esculentum Mill var. Santa Clara), sob doses crescentes de glyphosate e imazapyr. 


\section{Glyphosate}
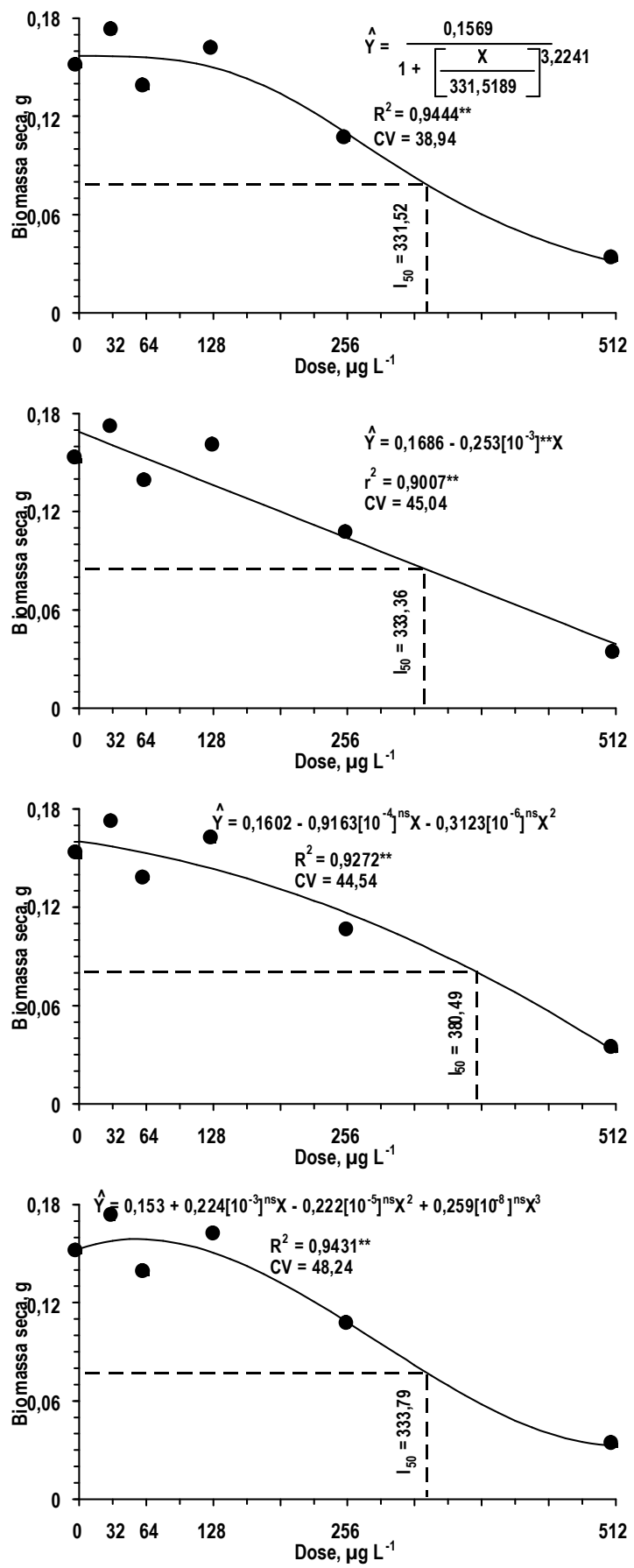

Imazapyr

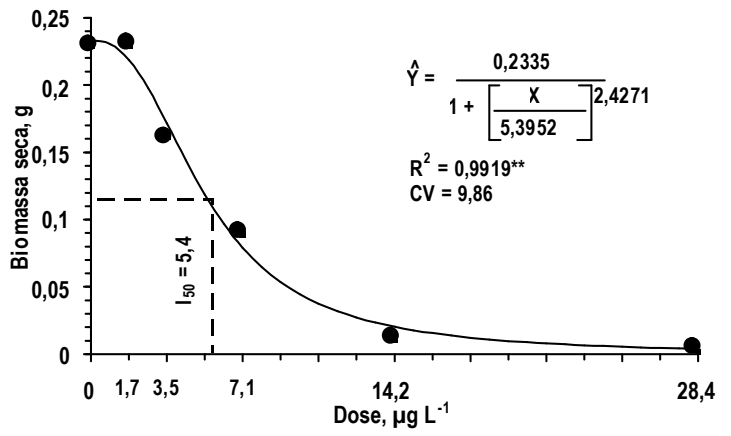

$\hat{Y}=0,1973 \cdot 0,8404\left[10^{-2}\right]^{\star * X}$

$r^{2}=0,7711^{* *}$

$\mathrm{CV}=45,49$
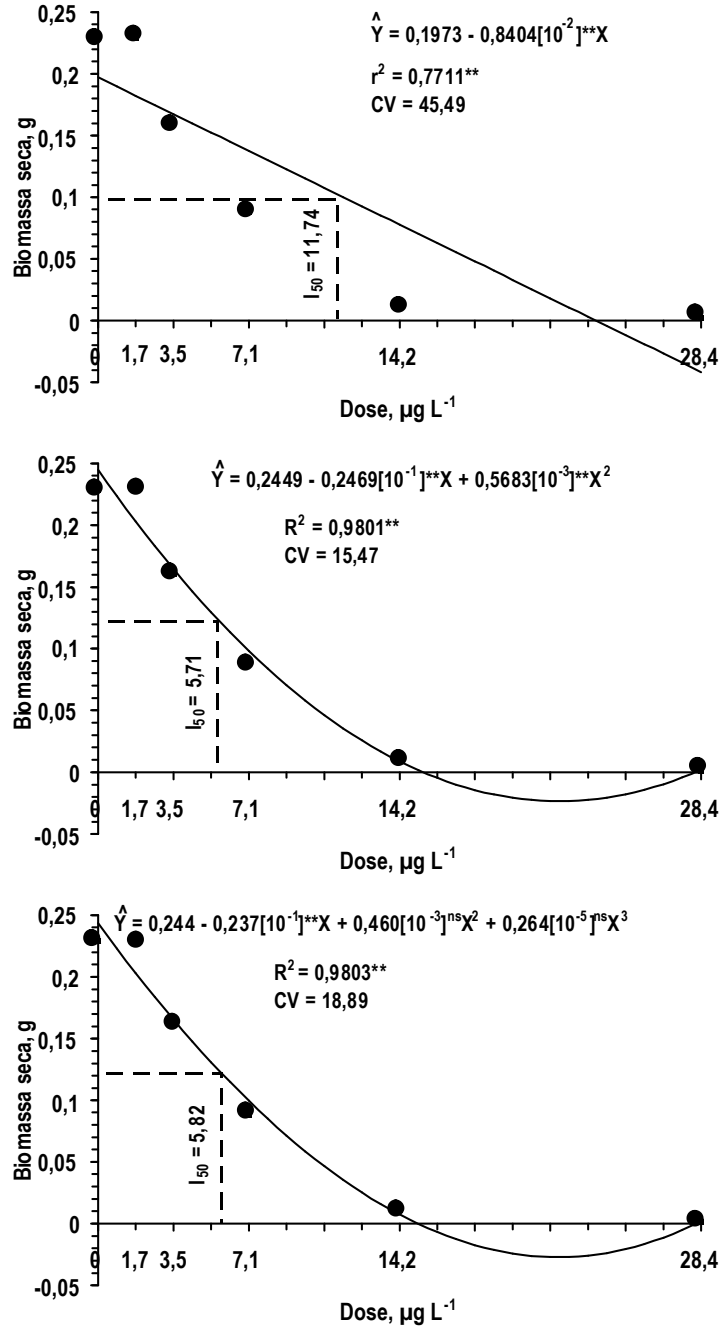

FIGURA 3. Curvas de dose-resposta da biomassa seca da raiz (BSR) do tomateiro (Lycopersicon esculentum Mill var. Santa Clara), sob doses crescentes de glyphosate e imazapyr. 


\section{Glyphosate}
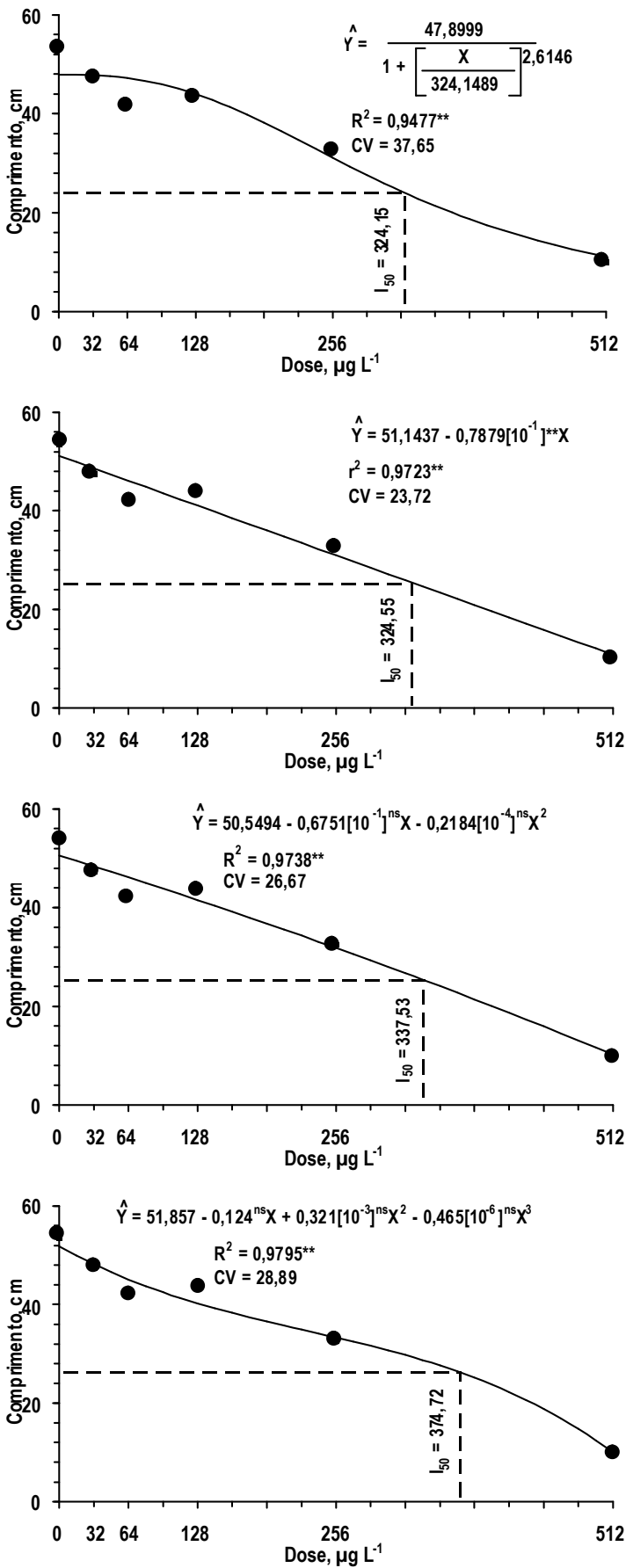

Imazapyr
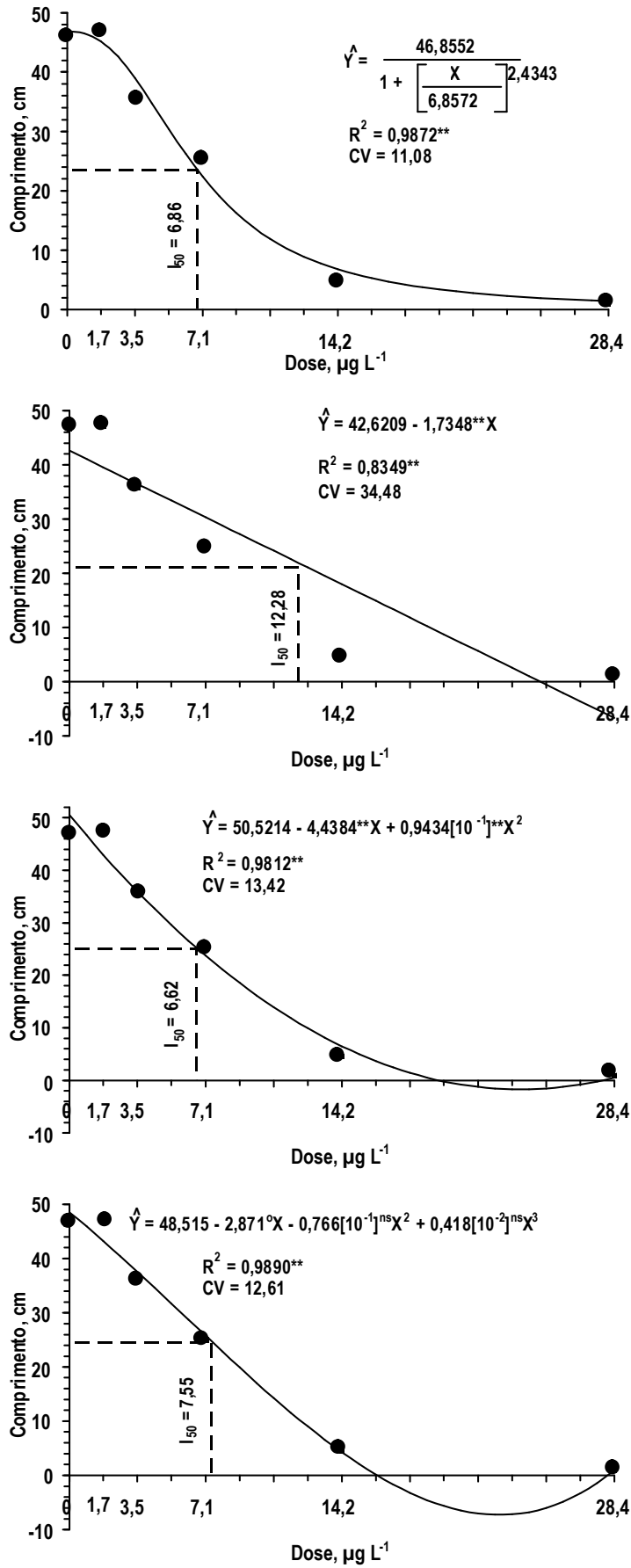

FIGURA 4. Curvas de dose-resposta do comprimento das raízes (CR) do tomateiro (Lycopersicon esculentum Mill var. Santa Clara), sob doses crescentes de glyphosate e imazapyr. 
Segundo Streibig (1988), quando a biomassa seca da planta é graficamente plotada contra as doses do herbicida, esta apresenta normalmente uma conformação sigmóide simétrica, sendo o limite superior da curva representado pela resposta da testemunha e o limite inferior pela resposta da planta às altas doses do herbicida, podendo esta ser zero. Ocasionalmente, plantas tratadas com doses muito baixas apresentam um crescimento que se excede à testemunha. Esse fenômeno, denominado "hormesis", ocorre em diversos herbicidas ou grupos de herbicidas, independentes do seu mecanismo de ação (Streibig, 1980) e foi observado apenas nos dados de biomassa seca para os dois herbicidas.

Nos modelos linear e quadrático, para o glyphosate; e linear, quadrático e cúbico, para o imazapyr, constata-se um rápido decréscimo da biomassa seca e do comprimento de raiz, já nas menores doses. Tais condições são pouco esperadas, uma vez que as doses escolhidas foram intercaladas (eq. 1), de tal forma que abrangessem quase todo o intervalo da curva teórica de resposta do bioindicador. De acordo com Seefeldt et al. (1995), para se admitir o desenvolvimento de uma equação que estime o melhor ajuste da curva doseresposta aos dados, a escolha das doses deve abranger toda a sequiência de resposta do bioindicador.

Os resultados obtidos para $\mathrm{I}_{50}$ apresentam variabilidade entre as funções e as variáveis analisadas, sendo esta mais expressiva para o imazapyr, o que indica que a estimativa desse índice depende da escolha da função e da própria variável. Considerando-se a expectativa de se obter o menor valor de $\mathrm{I}_{50}$, a função logística apresenta-se como a mais adequada para a estimativa desse índice, visto que a mesma, independente da variável analisada, estimou valores, quando não o menor, sempre próximo do menor índice de outra função. Observa-se ainda que o imazapyr apresentou os menores valores de $\mathrm{I}_{50}$, em todas as variáveis analisadas, indicando maior sensibilidade do bioindicador a esse produto. Pelo fato de ambos os produtos atuarem na síntese de aminoácidos, pode-se inferir que os menores valores de $\mathrm{I}_{50}$, apresentados no imazapyr, sejam decorrentes de uma rota metabólica no tomateiro, mais eficaz que a do glyphosate.

\section{LITERATURA CITADA}

BERKSON, J. A statistically precise and relatively simple method of estimating the bio-assay with quantal response based on the logistic function. J. Am. Stat. Assoc., v.48, p.565-599, 1953.

DRAPER, N.R., SMITH, H. An introduction to nonlinear estimation. In: Applied regression analysis. 2. ed., New York: John Wiley e Sons., 1981. p.458-535.

CORDEIRO, G.M., PAULA, G.A. Modelos de regressão para análise de dados univariados. IMPA, Rio de Janeiro, 1989. $353 p$.

FINNEY, D.J. Bioassay and the practice of statistical inference. Int. stat. rev., v.47, p.1-12, 1979.

FINNEY, D.J. Probit analysis. $3^{\mathrm{a}}$ ed., Cambridge: University Press, 1971.

FINNEY, D.J. Radioligand assay. Biometrics, v.32, p.721-740, 1976.

GAUSTON, D.R., VENUS, J.C. The biometry of plant growth. London: Edward Arnold, 1981. 307p.

HICKS, C.R. Qualitative and quantitative factors. In: HICKS, C.R. Fundamental concepts in the design of experiments. $2^{\mathrm{a}}$ ed., New York: Holt, Rinehart and Winston, Inc., 1973. p.123-152.

HOAGLAND, D.R., ARNON, D.I. The waterculture method for growing plants 
without soil. Berkeley, California, University of California College of Agriculture, Agricultural Experiment Station. December, p.1-39, 1938. (Circular, 347).

HOFFMANN, R., VIEIRA, S. Análise de regressão: uma introdução à econometria. $2^{a}$ ed., São Paulo: HUCITEC, 1983. 379p.

MARQUARDT, D.W. An algorithm for leastsquares estimation of nonlinear parameters, J. soc. indust. appl. math., v.11, p.431$441,1963$.

MOTULSKY, H.J., RANSNAS, L.A. Fitting curves to data using non-linear regression: a pratical and nonmathematical review. FASEB J., v.1, p.365-374, 1987.

NELDER, J.A. The fitting of a generalization of the logistic curve. Biometrics, v.17, p.89110, 1961.

NELDER, J.A., MEAD, R. A simplex method for function minimization. Comput. J., v.7, p.308-313, 1965.

NYFFELER, A., GERBER, H.R., HURLE, K. et al. Collaborative studies of dose-response curves obtained width different bioassay methods for soil-applied herbicides. Weed Res., v.22, p.213-222, 1982.

SAEG. Sistema para Análises Estatísticas; versão 7.1. Viçosa: Fundação Arthur Bernardes, 1997.

SEEFELDT, S., JENSEN, J.E., FUERST, E.P. Log-logistic analysis of herbicide doseresponse relationships. Weed Technol., v.9, p.218-227, 1995.

STEEL, R.G.D., TORRIE, J.H. Analysis of variance I: The one-way classification. In: Principles and procedures of statistics. With special reference to the biological sciences. New York: McGraw-Will Book Company, Inc., 1960. p.99-128.

STREIBIG, J.C. Herbicide bioassay. Weed Res., v.28, p.479-484, 1988.

STREIBIG, J.C. Models for curve-fitting herbicide dose response data. Acta agric. scand. Supll., v.30, p.59-64, 1980.

STREIBIG, J.C., RUDEMO, M., JENSEN, J.E. Dose-response curves and statistical models. In: STREIBIG, J.C., KUDSK, P. Herbicide bioassays. Florida: CRC Press, Inc, 1993. p.29-55. 